\title{
COLUMN
}

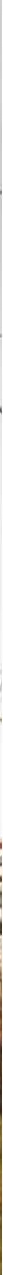

Assets managers in any electric, gas, or water utility face similar challenges when trying to meet their respective companies' obligations to the owners, operators, regulators, and the public affected by these assets

\section{Evidence-based \\ asset management}

T hose charged with managing assets in any electric, gas, or water utility face similar challenges when trying to meet their respective companies' obligations to the owners, operators, regulators, and the public affected by these assets.

In this column, the focus will be on both liquid-immersed distribution and pow- er transformers - key components in any network. With the onset of DER (Distributed Energy Resources) located at the grid edge, this will change the mode of system operation and will have an impact on managing the existing assets.

Asset management decisions require accurate information to produce a riskbased prioritization of investments to determine the physical state of any asset. In other words, one needs to sort out the candidates that meet the business objectives for repair (major or minor), refurbishment, replacement or disposal.

Some asset managers currently use a 'Health Index' for prioritising asset replacement. However, in many cases the index does not provide any indication 
The words "evidence-based" are commonly used to describe practices in medicine and healthcare industry, such as 'evidence-based medicine. However, the underlying principals do not change and can apply across most industries. The main concept being: when decisions are made, they are made based on the most up-to-date, solid and reliable scientific evidence. In this case, these are the decisions concerning the condition of individual assets.

EBAM is more than just a number, it is a continuous improvement process as suggested in Figure 1.

\section{Building confidence into a transformer condition assessment}

The recently published CIGRE Technical Brochure 761 (TB 761), Condition Assessment of Power Transformers [1] developed by CIGRE WG A2.49 aims to serve as a comprehensive guide to the development of transformer assessment indices for different purposes and discusses how to manage the issues listed above. It forms part of the key series of steps for transformer management outlined in CIGRE's Guide for Transformer Maintenance 445 (TB 445) [3]. It serves of how quickly the worst transformers on the list need to be actioned, nor do many of them provide any indication of the most appropriate action needed i.e. replace, repair or refurbish. To increase the confidence in the data, asset managers should consider using a process called evidence-based asset management (EBAM).

\section{Evidenced-based asset management (EBAM)}

Definition: An evidence-based practice is any practice that relies on scientific evidence for guidance and decision-making. Practices that are not evidence-based may rely on tradition, intuition, or other unproven methods.

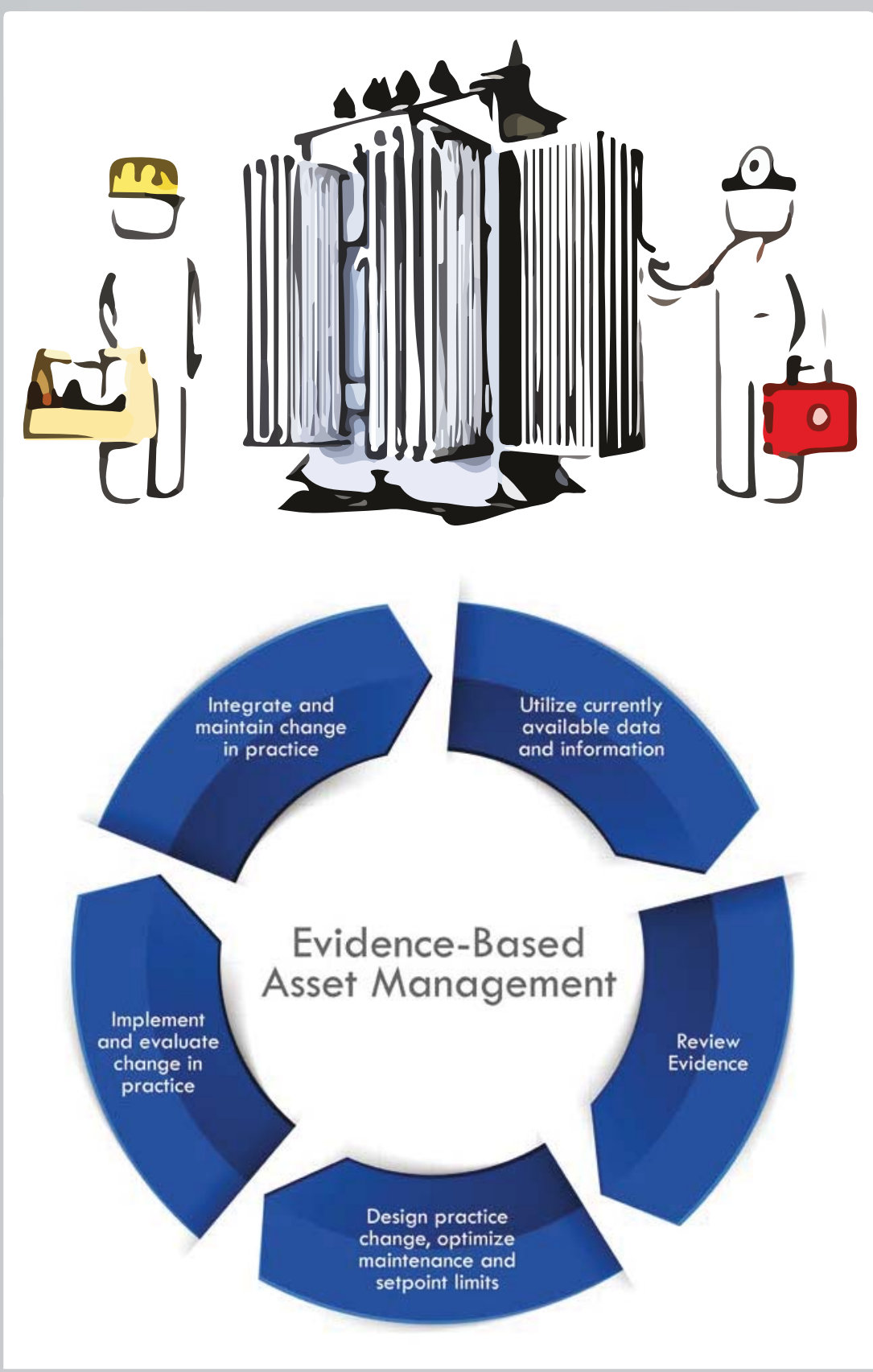

Figure 1. Elements in an EBAM structure 


\section{Asset management decisions require ac- curate information to produce a risk-based prioritization of investments to determine the physical state of any asset}

not only as a reference guide to transformer failure modes and suitable condition assessment techniques to manage the asset, but as a comprehensive guide to developing indices for different purposes to improve transformer management outcomes.

The TB is intended to be used for medium to long term asset planning. Transformer assessment indices are not intended to assist in short term/operational decision making. If a condition indicating imminent failure is detected during a transformer assessment, prompt action should be taken [1].

The guide points out that it is critical to determine the purpose of an assessment program answering the question "why" and obtaining 'buy in' from those who will either contribute to the effort or will

\section{The TB 761 provides the follow- ing for the readers:}

- A brief overview of transformer asset management and condition assessment requirements;

- Analysis of different types of indices, how they may be constructed, and their limitations;

- Guidance on dealing with missing or obsolete information, including examples;

- Detailed examples on developing different types of indices, including replacement, refurbishment, and repair indices; and

- Guidance on key transformer components that should be considered when building a transformer assessment index, along with suitable diagnostic techniques. use its output. I would refer the reader to my column in Volume 5, Issue 2 of Transformers Magazine, "The Future of Substation Monitoring" for a deeper insight [7].

Asset managers can use this model to begin building the process of utilizing data and information they currently have available. The process comprises of the following steps, taken from CIGRE TB 761 Guide of Transformer Condition Assessment [1].

- Step 1: Determine the purpose of the Transformer Assessment Index (TAI), and scoring method

- Step 2: Identify the failure modes to be included in the TAI

- Step 3: Determine how each failure mode will be assessed

- Step 4: Design a calibrated system for categorising failure modes (scoring matrix)

- Step 5: Calculate a TAI Score for each transformer

Asset managers have confidence in their decisions due to the proper collection and analysis of data and the appropriate selection of decision criteria. Furthermore, one needs to develop a method designed to consider missing or incorrect measurements. This is important from the point of view of the result (score) to the confidence in the score, and must be included in the planning and use of the assessment.

A question often asked is, what is the definition of medium or longer term. That is usually defined as a function of the purpose (the WHY) for the assessment process and business practices of the asset owner.

\section{Determining the end point of 'life'}

A common question is: how long will my transformer (or breaker, or switchgear) last before it meets its useful end of functional life or has become obsolete? An input to the answer is from the com- pany's policy on asset retirement (before it fails), its financial impact (is there any residual value of the asset?) and capital expense planning program.

In addition, one needs a deeper understanding and physical evidence, other than the traditional indirect condition data/information, that comes out of the methods suggested in TB 761.

A good example is the use of Furanic compounds dissolved in the oil and measured in a qualified laboratory. The unit pictured in Figure 2 represents the optimum point in time, during a factory tear down, to obtain paper samples from top, middle and bottom of the windings, and carry out a DP test, and compare against the lasted Furanic compound testing on the oil of this unit.

In another situation, actual DP testing of the paper was performed as a series

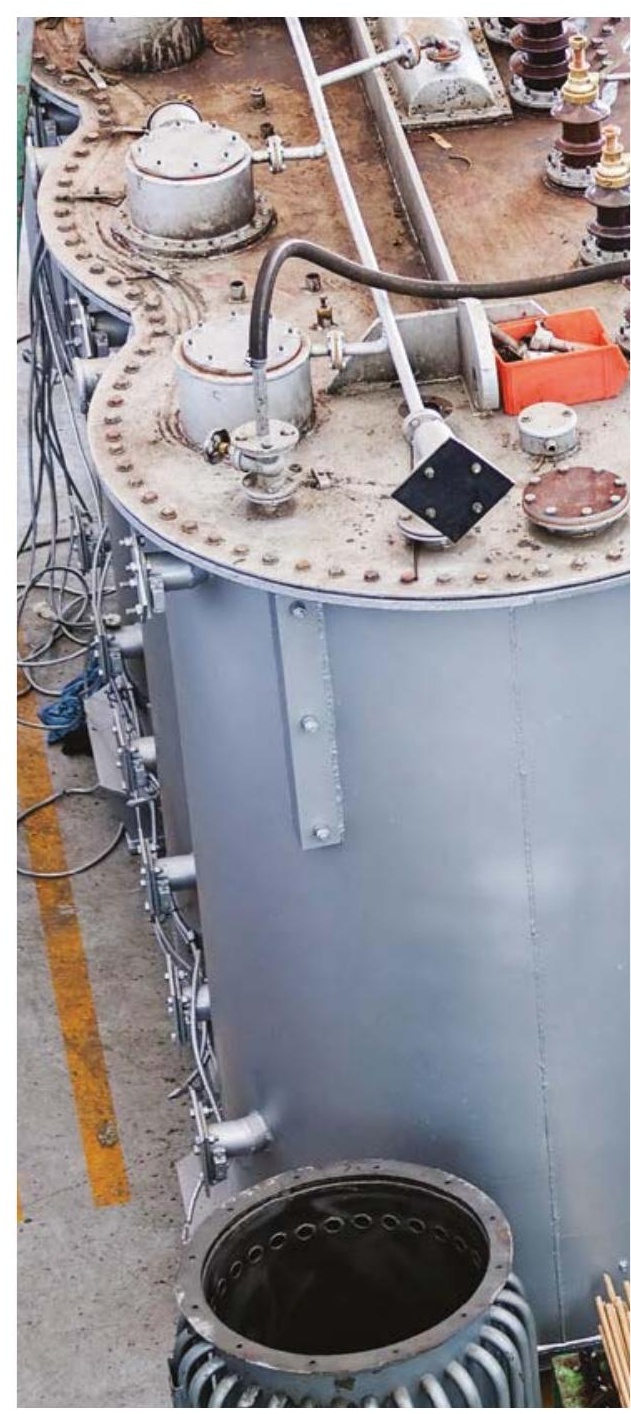


of rectifier transformers which came out of service after one unit failed in service. Paper samples were cut out away from the conductors at the top, bottom and some from the mid-section of the winding and subjected to the DP test procedure in a qualified laboratory.

The results were compared with 2-FAL concentration measured (Figure 3). NOTE that the paper in these designs was thermally upgraded Kraft paper (TUK). Normal Kraft paper would have had different results.

It is important to analyse both units that failed in service, as well as those that were removed (retired) from service before failure in order to gain further knowledge and test assumptions. CIGRE recently published TB 735 Transformer Post-Mortem Analysis [2] which details the process of carrying out a forensic inspection of these assets.

\section{A common question is: how long will my transformer last before it meets its useful end of functional life or has become obso- lete?}

The knowledge gained by an autopsy can then be applied to the existing fleet of that company where the same 'vintage' of equipment may still be in service.

Figure 4 is an example of a latent manufacturing or a design defect, that revealed itself too late. The forensic analysis of the evidence, along with the DGA trend and loading profile, can then be used on the remaining exiting units of the same vintage' to provide timing for removal from service for repair, refurbishment or replacement once the 'evidence' is understood.
Here is an example of following an EBAM model.

Recently a distribution utility started to gather evidence by carrying out forensic inspection on forty transformers rated $132 \mathrm{kV}$ and greater [4].

The inspection began to compare the evidence discovered with the symptoms of failure suggested from the data available (DGA, oil physical analysis, etc.) on units that either failed in service or have been removed from service for decommissioning. The cross-checking of results has generally validated and verified

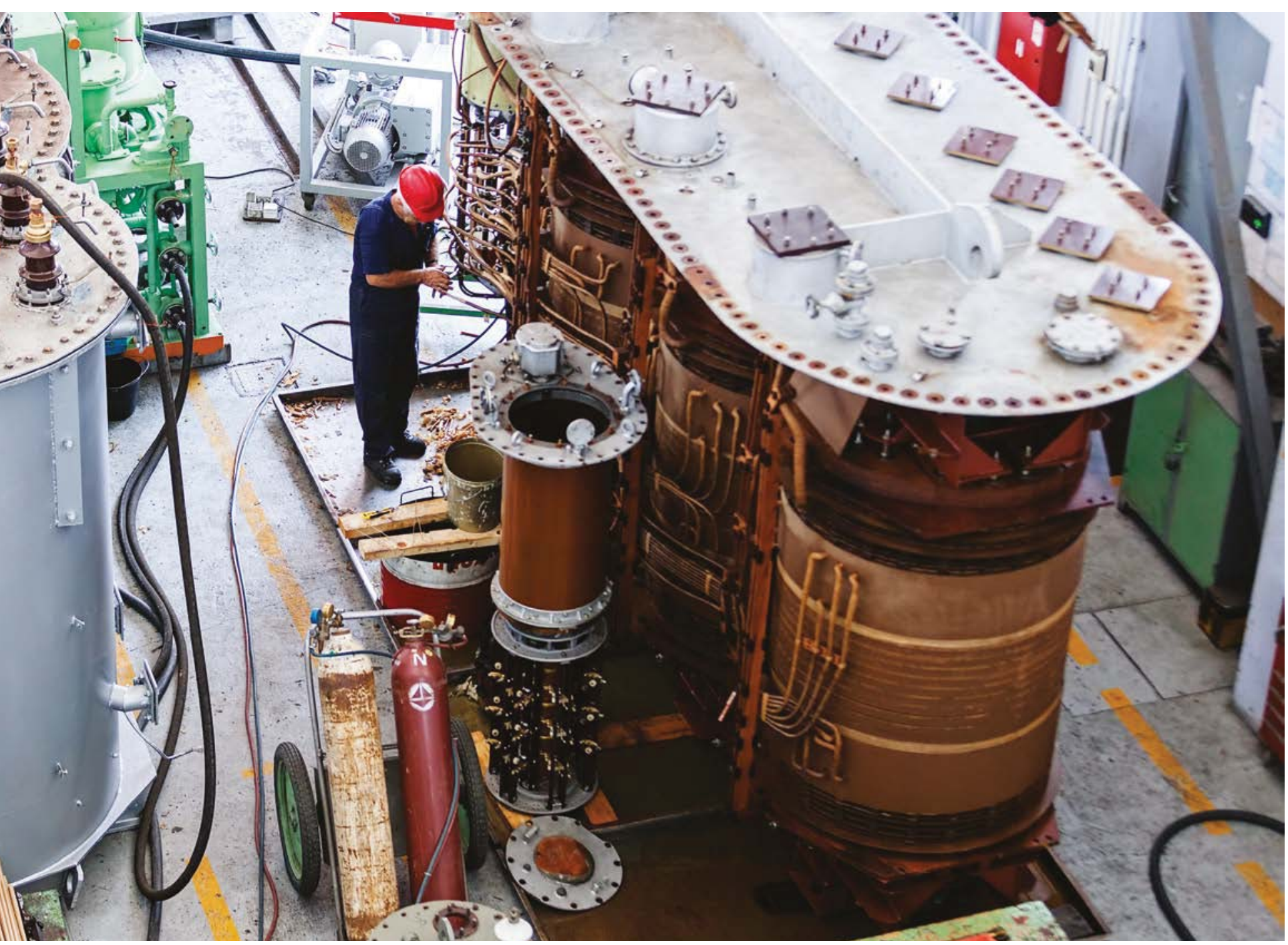

Figure 2. Factory tear down is the optimum time to gain knowledge on condition affecting the paper 


\section{An evidence-based practice is any practice that relies on scientific evidence for guid- ance and decision-making}

the adequacy and accuracy of these evidence but did highlight some anomalies that appeared and should be archived for future reference. The detailed data, photographs, and test results have not only benefitted the current professionals, but have left documented cases for a new generation of professionals to gain knowledge.

1) DGA analysis seemed to support industry experience: some units revealed thermal faults not clearly suggested by the analysis and four units presented clear signs of PD with only one of the four flagged (by DGA) was found to have this condition

2) Ten of the units were found to have severe sludge formation whereas the testing of the oil was not able to detect any presence whatsoever. Interfacial tension test did not provide any indication and acid levels were found to be only 'poor' in four of the ten.

3) Regarding predicted DP values calcu- lated from 2-FAL content in oil, there has been a variety of results when compared against actual measured DP results. When a comparison was made, the Chendong algorithm provided average differences between calculated DP and lowest measured DP of $16.1 \%$.

4) The inspections revealed that $61 \%$ of these transformers exhibited damage to core bolts or core bolt insulation caused by excessive and prolonged overheating. This evidence led to the utility changing their specifications for new units to be constructed using only banded or tapped yokes and limbs.

5) Visual inspections revealed that only $16 \%$ of the units had any main tank corrosion and $30 \%$ had cooler bank corrosion. However, a significant number (66 \%) displayed oil leakages of different sizes and magnitudes. The primary source was leakages from flange locations. Oil leakages, no matter the size or location, are a great concern as they provide the path for moisture and oxygen to enter the tank and can accelerate the loss of life of both the oil and solid insulation. They need to be fixed when discovered.

6) OLTCs exhibited deterioration of loose or displaced corona shields and pitted or worn contact surfaces. It was noted certain makes and models of OLTCs had these issues. These observations became part of the historical archive of facts.

\section{Conclusions reached from this experience to date}

- Help identify defects which could affect a full family or vintage of units still in service.

- Definition of future interventions for equipment of the same vintage exposed to similar service conditions (replacement versus refurbishment).

- Improvements of maintenance regimes with a final view of moving towards condition-based maintenance.

- The development of specifications for new equipment captures and addresses issues experienced in the fleet to reduce the risk of the same situation happening in the future. One item

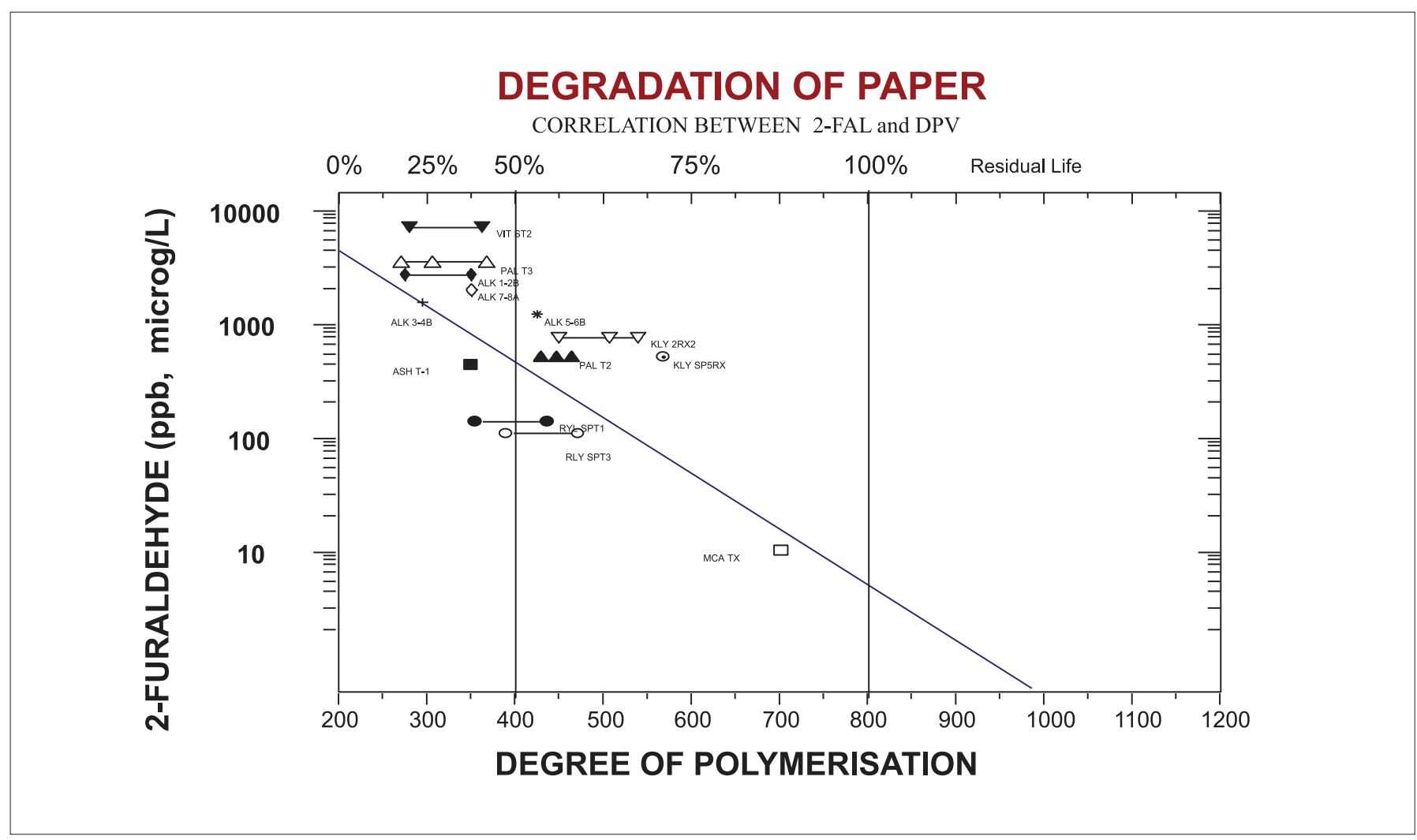

Figure 3. Comparison of 2-FAL and measured DP of paper samples taken from rectifier transformers removed from service [6] 
that fits in this narrative is the lubrication materials and methods for circuit breakers.

Applying principles of EBAM to asset management decisions generates huge savings for companies, up to tens of millions of dollars annually [5]. The four key areas that an EBAM can have the greatest impact on are:

- Lifecycle costing decisions;

- Maintenance tactics, such as preventive replacement strategies;

- Inspection policies, such as predictive maintenance and failure finding intervals;

- Resource requirements, such as establishing maintenance crew sizes.

Demonstrated in the examples above, EBAM can help asset managers to:

1. Find the optimum retirement ages of assets

2. Optimize maintenance and inspection frequencies

3. Establish the most economical preventive replacement intervals for components and hold the correct spare parts

4. Determine the best repair versus replacement decision policy

5. Make optimum condition-based maintenance decisions

\section{Bibliography}

[1] Technical Brochure 761, Condition Assessment for Power Transformers, CIGRE, April 2019

[2] Technical Brochure 735, Transformer Post-mortem Analysis, CIGRE, June 2018

[3] Technical Brochure 445, Guide for Transformer Maintenance, CIGRE, February 2011

[4] J. Quintana, D. Walker, I. Hunter, End of Life Evaluation of Power Transformers, paper number 0865, CIRED $25^{\text {th }}$ Conference, Madrid, Spain, June 3-6, 2019

[5] https://reliabilityweb.com/articles/ entry/evidence-based-asset-maagement-actionable-intelligence-in-theera-of-big-data

[6] B. Sparling, Assessing the Life of the Transformer, When Is It Time to "Pull the Plug"?, EUCI Conference, Vail CO, April 2000

[7] B. Sparling, Future of substation monitoring: It is not just the software, Transformer Magazine, vol. 5, issue 2, p.p. 64-68, April 2018

\section{Applying principles of EBAM to asset man- agement decisions generates millions in savings for companies}

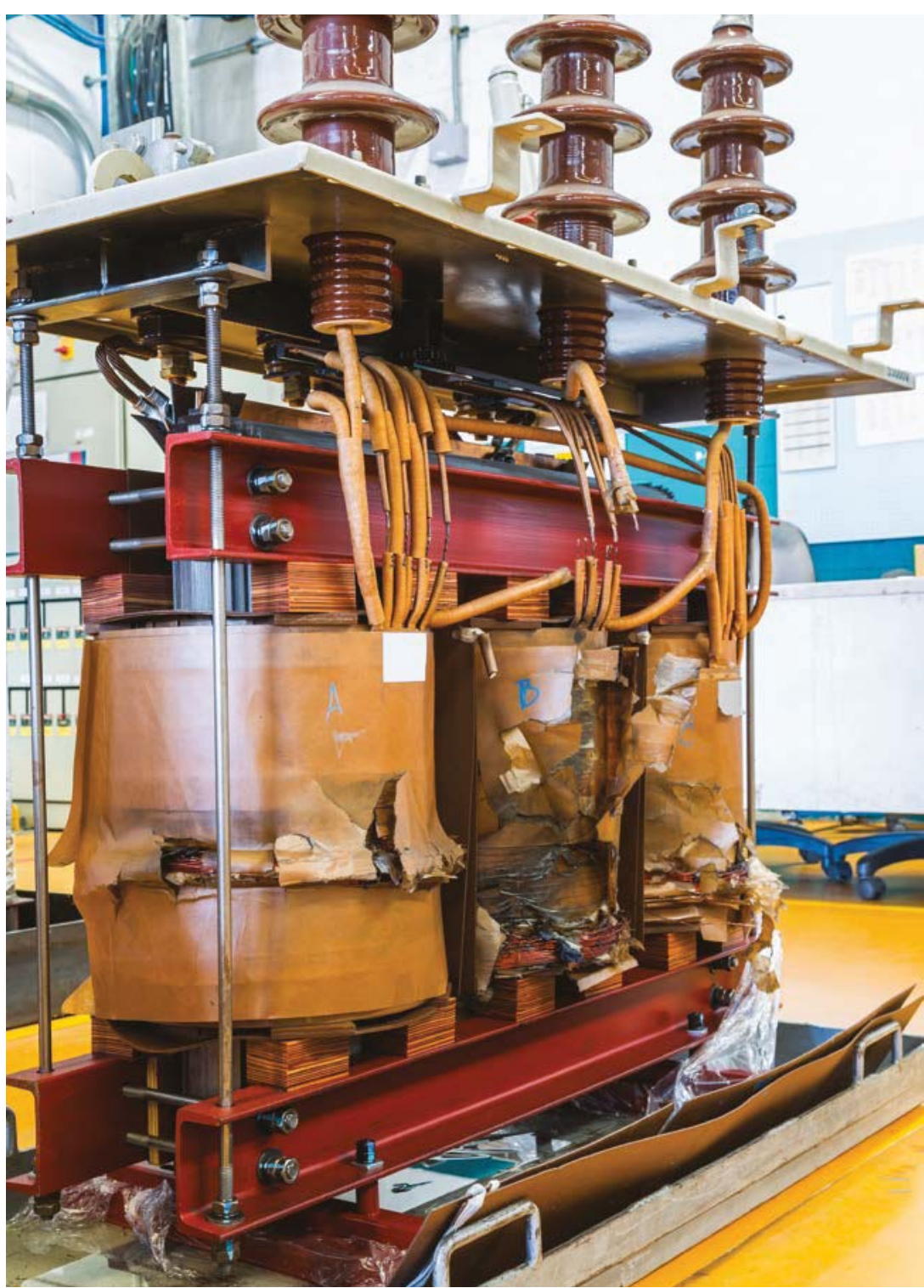

Figure 4. Potential latent manufacturing or design defect offers the chance to apply discoveries to other units of the same vintage

\section{Author}

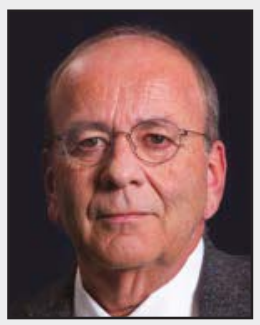

Brian D. Sparling, a Senior Member of IEEE, is a Senior Technical Advisor with Dynamic Ratings Inc. Brian has over twenty years of experience in the field of power and distribution transformers. For the last 28 years, he has been involved in all aspects of on-line monitoring and diagnostics and condition assessment of power transformers. He has authored and co-authored more than 33 technical papers on several topics dealing with the monitoring and diagnostics of transformers. He has worked on many guides and standards with the Canadian Electricity Association, IEEE Transformers Committee, and the CIGRÉ A2 Transformer committee. 